\title{
Cultivating mindfulness: evaluation of a community-based mindfulness program for Arabic-speaking women in Australia
}

\author{
Ilse Blignault ${ }^{1}$ (I) $\cdot$ Hend Saab $^{2} \cdot$ Lisa Woodland ${ }^{3}$ (D) Cathy O'Callaghan ${ }^{4}$ (I)
}

Accepted: 22 July 2021

(C) The Author(s) 2021

\begin{abstract}
Arabic-speaking communities in Australia underutilise mental health services. Previous research with Arabic-speakers recruited individually demonstrated that an Arabic Mindfulness Compact Disc (CD) was an acceptable and useful resource for this group. Subsequently, the $\mathrm{CD}$ was introduced as part of a 5 -week group mindfulness program for Arabic-speaking Muslim women. The intervention was delivered in a community setting by a bilingual (Arabic/English) psychologist with support from a bilingual multicultural health worker. The mixed-methods evaluation incorporated a pre-post study with a wait-list control group. An Arabic translation of the Depression Anxiety and Stress Scale (DASS21) was administered at baseline and program completion. Pre-post differences were tested using the sign test for paired samples (one-sided). Qualitative methods were used to evaluate the program's acceptability. After five weeks, the intervention group showed statistically significant improvement on all DASS2 1 subscales $(n=12, p<.001$ for depression and stress and $p<.01$ for anxiety). For the wait-list control group, only anxiety showed significant improvement $(n=8, p<.05)$. Qualitative analysis revealed how the women grew in their understanding of mindfulness concepts and mastered the different techniques, how they dealt with painful memories, how mindfulness practice assisted them in their daily lives, and how they related mindfulness to Islam. The in-language mindfulness intervention was shown to be culturally and spiritually relevant, and clinically effective. The group program incorporating the Arabic Mindfulness CD has potential for scaling up. Further research is required to investigate effectiveness for Arabic-speaking men with similar backgrounds.
\end{abstract}

Keywords Arabic $\cdot$ Migrant $\cdot$ Refugee $\cdot$ Muslim $\cdot$ Meditation $\cdot$ Stress

\section{Background}

Multicultural Australia is home to people from around the world (Jupp, 2001). At the 2016 Census of Population and Housing, 26\% of Australian residents were born overseas while another $21 \%$ of the population had one or both parents

Ilse Blignault

i.blignault@westernsydney.edu.au

1 Translational Health Research Institute, Western Sydney University, Locked Bag 1797, Penrith, NSW 2751, Australia

2 Multicultural Health Service, South Eastern Sydney Local Health District, PO Box 1614, Sydney, NSW 2001, Australia

3 Priority Populations, Population and Community Health, South Eastern Sydney Local Health District, PO Box 1614, Sydney, NSW 2001, Australia

4 South Eastern Sydney Research Collaboration Hub (SEaRCH), Centre for Primary Health Care and Equity, UNSW Sydney, Sydney, NSW 2052, Australia who were born overseas. Further, $21 \%$ of Australians reported speaking a language other than English at home. Arabic was the third most common language spoken (1.4\% of the total population), after English and Mandarin (Australian Bureau of Statistics [ABS], 2017). The Arabic-speaking community in Australia comprises individuals from various countries and religions and both long-standing residents and new arrivals, including refugees and others with refugee-like backgrounds (Jupp, 2001; Khawaja \& Khawaja, 2016a).

Mental health issues are common in migrant communities generally and increased among refugees and asylum seekers (Minas et al., 2013; Silove, 2003; Slewa-Younan et al., 2017). In common with many other minority communities in Australia, Arabic-speakers underutilise mental health services (Kayrouz et al., 2015; McDonald \& Steel, 1997; Slewa-Younan et al., 2015; Steel et al., 2006), preferring to rely on family, family doctors, traditional healers and religious leaders (Kayrouz et al., 2015; Tobin, 2000; Youssef \& Deane, 2006). The Arabic Mindfulness Compact Disc (CD) was developed to address an identified need for a culturally-tailored, evidence-based resource 
that can be used with, and by, members of this heterogeneous community (Blignault et al., 2019).

Mindfulness is a form of meditation that derives from Buddhist practice. It has been popularised in the West through the work of Jon Kabat-Zinn (2003) who describes it as "the awareness that emerges through paying attention, on purpose, in the present moment, and non-judgmentally to the unfolding of experience moment by moment" (p. 145). While some authors emphasise its Buddhist origins (e.g. Kabat-Zinn, 2003), others find resonance with religious and spiritual traditions such as Islam (Mirdal, 2012; Thomas et al., 2017) and Christianity (Rosales \& Tan, 2017; Trammel, 2015), or take a secular approach (Baer, 2015).

The past two decades have seen exponential growth in interest in the application of mindfulness and meditation practice in medicine and mental health care globally (Van Dam et al., 2018). Research demonstrates the benefits of Mindfulness-Based Interventions (MBIs) for patients with mental and physical health conditions (Creswell, 2017) and for non-clinical populations (Janssen et al., 2018). MBIs, such as mindfulness-based stress reduction (MBSR) and mindfulness-based cognitive therapy (MBCT), can significantly alleviate depression, anxiety and stress and improve physical and psychological functioning (Creswell, 2017; Janssen et al., 2018). Brief MBIs, totalling up to $100 \mathrm{~min}$ ' practice per week and lasting up to four weeks, can also be beneficial (Howarth et al., 2019).

While there is emerging research on mindfulness in Middle Eastern countries (Damra et al., 2014; Güldal \& Satan, 2020; Pigni, 2010; Thomas, Raynor, \& Bahussain, 2016b; Thomas, Raynor, \& Bakker, 2016a) there are few studies with Arabic speakers who constitute a minority group in Western countries. The situation is not unique to this language group (Russell et al., 2019) or to Australia (DeLuca et al., 2018). In the United States, there is a lack of diversity focus in mindfulness research, with ethnic minorities under-represented in studies assessing mental health outcomes and few reports of culturally-adapted interventions (DeLuca et al., 2018).

The Arabic Mindfulness $C D$ is a cultural adaptation of Mindfulness Skills Volume 1 - Learn "mindfulness" skills produced by Dr. Russ Harris (Blignault et al., 2019). The 60-min educational CD contains five tracks. The adaptation process involved five steps: (1) initial translation into formal Arabic by the second author, a bilingual (Arabic/English) psychologist who also amended Track 1 to emphasise the cultural and spiritual relevance of mindfulness and mindfulness practice to the Arabic culture; (2) review by a bilingual mental health nurse and five bilingual community members; (3) review and modification by a nationally accredited translator; (4) re-checking by the bilingual mental health clinicians and community members; and (5) minor editing by the accredited translator.
The first phase of its evaluation involved a pre-post study (Blignault et al., 2019). The 70 participants were Arabicspeaking adults living in south-east Sydney who were given the $\mathrm{CD}$ as a self-management resource. Most were Lebaneseborn women and men who had been in Australia for over 15 years. Both Muslims and Christians were included. The Arabic Mindfulness CD was shown to be culturally acceptable and effective in reducing levels of psychological distress as measured by the Kessler Scale (K10) and the Depression, Anxiety and Stress Scale (DASS21). Following those promising findings, it was proposed that the Arabic Mindfulness $\mathrm{CD}$ be further tested in a group setting and in different geographic locations, as well as with newly-arrived Arabicspeakers with refugee-like backgrounds. This paper reports on the second phase of the evaluation.

\section{Methods}

\section{Setting}

The current study was conducted in Wollongong, a large city located about $100 \mathrm{~km}$ to the south of Sydney. Project partners were the South Eastern Sydney Local Health District (SESLHD) Multicultural Health Service, SESLHD Mental Health Service, Illawarra Shoalhaven Local Health District Multicultural Health Service, Illawarra Multicultural Services and Western Sydney University. Illawarra Multicultural Health Services provided the venue for the group sessions. This non-government organisation provides a range of programs to meet the needs of new arrivals and migrant communities who are socially and/or financially disadvantaged with low English language proficiency.

\section{Target Group}

Given that the group program sought to attract Arabicspeaking community members newly arrived to Australia, gender-specific groups were considered more likely to be culturally acceptable than mixed groups. The target group was Arabic-speaking women who had lived in Australia for 6 months or longer and were 18-65 years old (the age group served by adult community mental health services).

\section{Intervention}

The Mindfulness Program for Arabic-speaking Women was delivered at Illawarra Multicultural Services between August and November 2016. Participants were required to attend group sessions once a week for five weeks and to listen to specified tracks of the Arabic Mindfulness CD at least twice each week. The weekly sessions were facilitated in Arabic language by a bilingual (Arabic/English) psychologist with 
support from a bilingual multicultural health worker. Free child minding was provided.

Table 1. Overview of the Mindfulness Program for Arabic-speaking Women

\section{Group session 1: Introduction to Mindfulness \\ Content: What is mindfulness and definitions of mindfulness; Balance of stressors and resources; Origins of mindfulness, and how this relates to spiritual and cultural beliefs and practices; Auto-pilot vs. being mind- ful; Daily activities where you need to be mindful; Benefits of mind- fulness.}

Mindfulness practice: Grounding, Mindfulness of the Breath and Leaves on a Stream.

Core resources: CD; Participant Handbook.

Handouts: What is mindfulness; Automatic pilot.

Homework: CD Track 1 Introduction; Track 2 Mindfulness of the Breath; Track 4 Leaves on a Stream.

\section{Group session 2: Contact with the Present Moment}

Content: What is stress; Stressors and the stress response; Identifying your own stress symptoms including bodily sensations, emotions and thoughts; Good and bad stress; Signs of stress overload; How mindfulness can help to reduce stress; Tips to practice living in the moment; STOPP (Stop, Take a breath, Observe, Pull back - Put in some perspective, Practice what works - Proceed).

Mindfulness practice: Mindfulness of the Breath.

Handouts: Thoughts, feelings and behaviours; STOPP technique.

Homework: CD Track 2 Mindfulness of the Breath; Track 4 Leaves on a Stream.

Group session 3 - The Observing Self

Content: Judgement, self-judgement and non-judgement; Poisoned parrot exercise which teaches participants to notice and learn about the detrimental impact of negative self-talk; Link between thoughts, feelings and behaviours; Self observing - thoughts are not facts.

Mindfulness practice: Leaves on a Stream and The Observing Self.

Handout: The Poisoned Parrot.

Homework: CD Track 4 Leaves on the Stream; Track 5 The Observing Self.

Group Session 4 - Loving Kindness and Self-compassion.

Content: Loving kindness; Self-compassion; Three elements of self-compassion (1) Self -kindness vs. self-judgement, (2) Common humanity vs. isolation, (3) Mindfulness vs. over-identification; Benefits of self-compassion; Compassion meditations.

Mindfulness practice: Leaves on a Stream and The Observing Self.

Homework: CD Track 4 Leaves on a Stream; Track 5 The Observing Self.

Group session 5 - Mindfulness of Emotions.

Content: Physical and psychological pain; Modifying suffering; How mindfulness can help to reduce the suffering associated with psychological pain; Sitting with painful emotions by applying self-compassion and loving kindness practices; Breathing exercises; Three ways to help sitting with emotions (1) Observe your emotions, (2) Validate your emotions (3) Focus on the present.

Mindfulness Practice: Leaves on a Stream and Mindfulness of Emotions. Handout: Mindfulness of emotions.

Homework: CD Track 3 Mindfulness of Emotions.
Program content, which was organised around the $\mathrm{CD}$ as the core component, was informed by previous experience with this target group (Blignault et al., 2019). Table 1 provides a program overview. At the first group session each woman received a 43-page participant handbook that included information and worksheets. Extra handouts were provided as the course progressed. Most program material (PowerPoint slides, handbook and handouts) was in English. During the sessions, the bilingual facilitator spoke to the material and participants made notes in Arabic. Quotes from the Quran were introduced frequently by both facilitator and participants.

\section{Evaluation}

\section{Design}

The mixed-methods evaluation incorporated a pre-post study with a wait-list control group (see Fig. 1). Interested women were invited to attend an information session at which they received the Participant Information Sheet and Consent Form and had the opportunity to ask questions. Those who agreed to take part in the study were randomly allocated into two groups: an intervention group who began the program one week after the information session (Group 1) and a wait-list control group who began the program nine weeks later (Group 2). The start of the second program was determined by school holidays and venue and facilitator availability. Both groups were invited to a combined follow-up session held one week after the second program concluded.

Data were collected at the information session (baseline, Week 0), at the end of the first group program (Week 5) and at the end of the second group program (Week 13, Group 2 only). Additionally, participants were asked to keep a record of their home practice. A follow-up phone call roughly six months post-program provided the opportunity to ask additional background questions.

\section{Recruitment}

Phase 1 of the research indicated that word-of-mouth was particularly effective in recruiting members of the Arabicspeaking community so this method was employed, along with a flyer in Arabic and English that was distributed by mail, email and social media. The program was promoted through a range of health and community service providers including Arabic-speaking GPs, English-language program providers, and local social and religious groups.

\section{Measures}

A 3-part questionnaire, available in Arabic and English, was administered at recruitment. Sociodemographic items included: Age, Gender, Postcode, Country of birth, Years of 


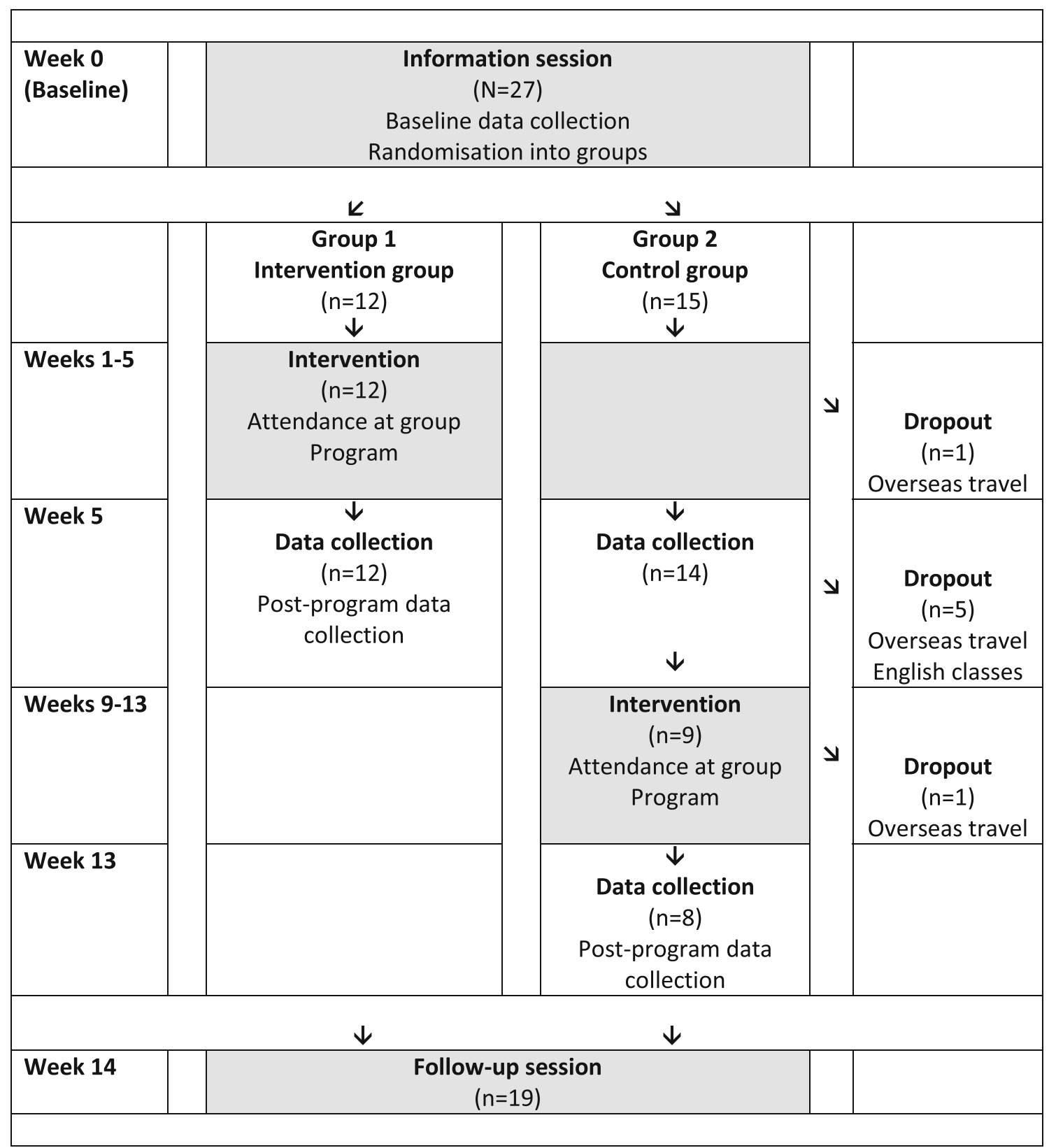

* Numbers in brackets refer to the number of participants engaged in that study component.

Fig. 1 Study implementation and evaluation

residence in Australia, Language spoken at home, Religion and Education. The second section asked about health professionals seen in the last four weeks: GP, Psychologist, Psychiatrist, Counsellor, Emergency Department or Hospital, and Other health professional. The final section comprised eight statements designed to assess knowledge of and attitudes toward mindfulness, with participants invited to show their agreement using a 5-point Likert scale: strongly disagree - strongly agree. A similar questionnaire (minus the sociodemographic items and with an extra question asking participants if they had shared the Mindfulness CD with friends or family) was administered at the end of the program. The mental health outcome measure employed was an Arabic translation of the DASS21, a 21-item questionnaire with subscales designed to measure depression, anxiety and stress (Lovibond \& Lovibond, 1995a), which was developed, validated and distributed by the New South Wales Transcultural Mental Health Centre (Moussa et al., 2016).

Home practice log sheets contained spaces for recording when participants listened to each track on the $\mathrm{CD}$, for how long, and any comments. These were collected at the last group session, and comments in Arabic translated into English for analysis by the bilingual multicultural health worker. The facilitator kept a record of group attendance and wrote extensive notes in English recording participants' responses and comments during the group sessions and 
afterwards. Over the course of the program, the high level of war-related trauma became apparent. At a follow-up phone call from the facilitator, when a trusting relationship had been established, all participants were asked about their visa status, if they had experienced or witnessed violence in their country of origin or during the journey to Australia, and if they wanted additional support to deal with any lasting distress.

\section{Analysis}

For quantitative analysis, data from the questionnaire and the DASS21 were entered into an Excel Spreadsheet with translations from Arabic to English where necessary. DASS21 subscales scores were categorised according to the manual (Lovibond \& Lovibond, 1995b): depression as normal (0-4), mild (5-6), moderate (7-10), severe (11-13), or extremely severe (14+); anxiety as normal (0-3), mild (4-5), moderate (6-7), severe (8-9), or extremely severe (10+); and stress as normal (0-7), mild (8-9), moderate (10-12), or severe (1316). The number of occasions of home practice was calculated from $\log$ sheet entries.

Descriptive statistics were used to summarise the sociodemographic data, trauma experiences and health professionals seen before and during the program, and compliance with the program protocol (number of group sessions attended and home practice occasions reported). Baseline and post-program comparisons focussed on: (1) knowledge and acceptability of mindfulness as assessed on the Likert-scale questions and (2) levels of depression, anxiety and stress. Differences in DASS21 scores across time for the intervention and wait-list control groups were tested for statistical significance using the nonparametric sign test for paired samples (one-sided), as the data were not normally distributed. The null hypothesis was that the median difference would be zero, while the alternative hypothesis was that participants would show improvement post-intervention. A $p$ value of 0.05 was taken to indicate a significant result.

For qualitative analysis, data and comments from the participants' log sheets were entered into another Excel spreadsheet. Preliminary analysis was guided by the following questions: How did participants experience mindfulness practice? What were the results? What were the difficulties? How did they apply mindfulness in everyday day life and how did it help them (or not)? Emerging findings were grouped into general themes. Notes taken by the facilitator during followup phone calls were also scrutinised. Both sources of data were examined for comments relating to the group setting and sharing the Arabic Mindfulness CD. Manual thematic analysis (Green \& Thorogood, 2018) was completed by the fourth author, who used an inductive approach to generate codes which were then categorised and cross-checked with the bilingual multicultural health worker. Themes were finalised through discussion with the other authors.

\section{Ethics}

The SESLHD Human Research Ethics Committee approved the study protocol. Informed consent was obtained at the information session where the facilitator explained the intervention and the evaluation component, went through the Participant Information Sheet (available in Arabic and English) and answered any questions. Translation of the information and consent forms and data collection tools was undertaken by nationally accredited translators.

Participants who indicated a high level of psychological distress at recruitment, or at any point throughout the study, were offered support and referral to the mental health service or a private health professional (bilingual GP, psychologist or psychiatrist) as required. The study was monitored by a steering committee which included the first three authors.

\section{Findings}

Study findings are organised under five main headings: (1) Study participants; (2) Program adherence; (3) Pre- and post-study comparisons; (4) Experience of the Arabic Mindfulness CD; and (5) Social considerations.

\section{Study Participants}

Of the 27 women who attended the information session, enrolled in the study and provided baseline data; six later decided not to proceed. All six had been allocated to Group 2: some arranged overseas travel during the study period while others found they had conflicting English classes. Another Group 2 participant started the program but did not attend the final two sessions due to overseas travel. The remaining 20 women started and completed the 5-week program: 12 participating in the first group and 8 in the second group. Baseline data comparisons showed that the women who completed the program and the others shared similar backgrounds including length of time in Australia, understanding of mindfulness, and levels of depression, anxiety and stress.

\section{Sociodemographic Characteristics}

All 20 women were Muslim. Sixteen were aged between 26 and 55 years. Eleven were born in Iraq, 4 in Lebanon, 3 in Syria and 2 in Libya. Eleven had lived in Australia for under four years; three for over 11 years. At home 15 spoke Arabic, 
4 spoke Arabic and English, and 1 spoke Kurdish. Nine had post-school qualifications.

\section{Trauma Experiences}

Data collected from 18 of the participants revealed a history of war-related trauma. Eight were on refugee visas and and four on asylum/protection visas. Fifteen had personally experienced trauma and another three had witnessed trauma. In the group sessions and when talking privately with the facilitator, some of the women also spoke about domestic violence along with other problems experienced in the settlement period.

\section{Health Professionals Seen}

At baseline (i.e the information session held before the study) and again at the program's end (Group 1 in Week 5 and Group 2 in Week 13), most women reported visiting a GP during the previous month. Seeing a mental health professional was uncommon; at both times, only five reported seeing psychiatrist, psychologist or counsellor.

\section{Program Adherence}

Participants practiced mindfulness in each of the weekly group sessions. They were also asked to practice at home at least twice between sessions, giving a total of three times a week. Most participants achieved this:

- 12 attended all 5 group sessions, 6 attended four sessions, and 2 attended three sessions.

- 15 logged home practice every week, 4 for four weeks, and 1 for three weeks.

Over the whole program, the number of occasions of home practice ranged from 3 to 15 with a mean of 10.7. Total occasions of mindfulness practice (in the group and at home) ranged from 7 to 20 , with a mean of $15.2 \pm 3.6$, median of 15.5 and mode of 14.

\section{Pre and Post-Study Comparisons}

\section{Mindfulness Knowledge and Attitudes}

At baseline each of the questions assessing knowledge of and attitudes toward mindfulness received a few 'Don't know' responses. Post-program this was not the case. Although they held mixed views on whether mindfulness was a form of meditation (11 agreed and 9 disagreed) or a type of physical activity (6 agreed and 14 disagreed), all 20 participants agreed that mindfulness uses the breath to improve concentration and that it offers practical strategies to reduce stress. All agreed that mindfulness was compatible with their existing cultural and religious practices and that it fitted in with their way of life (19 strongly agreed with each statement). Table 2 shows all responses.

Table 2 Responses to knowledge and attitude questions for all participants $(N=20)$

\begin{tabular}{|c|c|c|}
\hline \multirow[t]{2}{*}{ Statement } & Baseline & Post-program \\
\hline & $\%$ & $\%$ \\
\hline
\end{tabular}

1. Mindfulness is a form of meditation

$\begin{array}{lcccc}\text { Strongly Agree } & 0 & 0 & 8 & 40\end{array}$

$\begin{array}{lllll}\text { Agree } & 14 & 70 & 3 & 15\end{array}$

$\begin{array}{lllll}\text { Neither agree nor disagree } & 0 & 0 & 0 & 0\end{array}$

$\begin{array}{lllll}\text { Disagree } & 0 & 0 & 1 & 5\end{array}$

$\begin{array}{lllll}\text { Strongly Disagree } & 0 & 0 & 8 & 40\end{array}$

Don't know $\quad 6 \quad 30 \quad 000$

2. Mindfulness is about focussing on the past and the future

$\begin{array}{lllll}\text { Strongly Agree } & 2 & 10 & 4 & 20\end{array}$

$\begin{array}{lllll}\text { Agree } & 9 & 45 & 0 & 0\end{array}$

$\begin{array}{lllll}\text { Neither agree nor disagree } & 0 & 0 & 0 & 0\end{array}$

$\begin{array}{lllll}\text { Disagree } & 5 & 25 & 1 & 5\end{array}$

$\begin{array}{lllll}\text { Strongly Disagree } & 2 & 10 & 15 & 75\end{array}$

$\begin{array}{lllll}\text { Don't know } & 2 & 10 & 0 & 0\end{array}$

3. Mindfulness uses the breath to improve concentration

$\begin{array}{lllll}\text { Strongly Agree } & 5 & 25 & 11 & 55\end{array}$

$\begin{array}{lllll}\text { Agree } & 11 & 55 & 9 & 45\end{array}$

$\begin{array}{lllll}\text { Neither agree nor disagree } & 0 & 0 & 0 & 0\end{array}$

$\begin{array}{lllll}\text { Disagree } & 2 & 10 & 0 & 0\end{array}$

$\begin{array}{lllll}\text { Strongly Disagree } & 0 & 0 & 0 & 0\end{array}$

$\begin{array}{lllll}\text { Don't know } & 2 & 10 & 0 & 0\end{array}$

4. Mindfulness is a type of physical activity

$\begin{array}{lllll}\text { Strongly Agree } & 1 & 5 & 3 & 15 \\ \text { Agree } & 9 & 45 & 3 & 15\end{array}$

$\begin{array}{lllll}\text { Neither agree or disagree } & 2 & 10 & 0 & 0\end{array}$

$\begin{array}{lllll}\text { Disagree } & 5 & 25 & 5 & 25\end{array}$

$\begin{array}{lllll}\text { Strongly disagree } & 1 & 5 & 9 & 45\end{array}$

$\begin{array}{lllll}\text { Don't know } & 2 & 10 & 0 & 0\end{array}$

5. Mindfulness is compatible with existing cultural and religious practices

$\begin{array}{lllll}\text { Strongly Agree } & 9 & 45 & 19 & 95\end{array}$

$\begin{array}{lllll}\text { Agree } & 7 & 75 & 1 & 5\end{array}$

$\begin{array}{lllll}\text { Neither agree nor disagree } & 1 & 5 & 0 & 0\end{array}$

$\begin{array}{lllll}\text { Disagree } & 0 & 0 & 0 & 0\end{array}$

$\begin{array}{lllll}\text { Strongly disagree } & 0 & 0 & 0 & 0\end{array}$

$\begin{array}{lllll}\text { Don't know } & 3 & 15 & 0 & 0\end{array}$

6. I would be willing to learn new ways of improving my wellbeing through Mindfulness

$\begin{array}{lllll}\text { Strongly Agree } & 13 & 65 & 20 & 100\end{array}$

$\begin{array}{lllll}\text { Agree } & 5 & 25 & 0 & 0\end{array}$

$\begin{array}{lllll}\text { Neither agree nor disagree } & 0 & 0 & 0 & 0\end{array}$

$\begin{array}{lllll}\text { Disagree } & 0 & 0 & 0 & 0\end{array}$

Strongly Disagree $\quad 0 \quad 0000$

$\begin{array}{lllll}\text { Don't know } & 2 & 10 & 0 & 0\end{array}$

$\begin{array}{lllll}\text { 7. Mindfulness fits in with my way of life } & & & \\ \text { Strongly Agree } & 1 & 5 & 19\end{array}$

$\begin{array}{lllll}\text { Agree } & 13 & 65 & 1 & 5\end{array}$

$\begin{array}{lllll}\text { Neither agree nor disagree } & 1 & 5 & 0 & 0\end{array}$

$\begin{array}{lllll}\text { Disagree } & 2 & 10 & 0 & 0\end{array}$

$\begin{array}{lllll}\text { Strongly disagree } & 0 & 0 & 0 & 0\end{array}$

$\begin{array}{lllll}\text { Don't know } & 3 & 15 & 0 & 0\end{array}$

8. Mindfulness offers practical strategies to reduce stress

$\begin{array}{lllll}\text { Strongly Agree } & 8 & 40 & 18 & 90\end{array}$

$\begin{array}{lllll}\text { Agree } & 7 & 35 & 2 & 10\end{array}$

$\begin{array}{lllll}\text { Neither agree nor disagree } & 1 & 5 & 0 & 0\end{array}$

$\begin{array}{lllll}\text { Disagree } & 1 & 5 & 0 & 0\end{array}$

$\begin{array}{lllll}\text { Strongly Disagree } & 0 & 0 & 0 & 0\end{array}$

$\begin{array}{lllll}\text { Don't know } & 3 & 15 & 0 & 0\end{array}$ 


\section{Depression, Anxiety and Stress}

Scores on the DASS2 1 subscales on the different measurement occasions (at baseline and Week 5 for Group 1 and at baseline, Week 5 and Week 13 for Group 2) are shown in Table 3. Both groups showed improvement over time. Group 2, who served as a wait-list control at Week 5, showed most improvement at Week 13 after completing the program.

At baseline, seven of the twelve participants in Group 1 scored as severe/extremely severe on depression, ten scored as severe/extremely severe on anxiety, and nine scored as severe/ extremely severe on stress. Post-program (Week 5), no-one scored as severe/extremely severe on depression or stress, and only three scored as severe/extremely severe on anxiety (Table 3). Improvement on all three DASS21 subscales was statistically significant $(p<.001$ for depression and stress, $p<.01$ for anxiety) (Table 4 ).

At baseline, four of the eight participants in Group 2 scored as severe/extremely severe on depression, anxiety and stress. At Week 5, three scored as severe on depression and stress, and two scored as severe/extremely severe on anxiety (Table 3). From baseline to Week 5, only the improvement in anxiety was statistically significant $(\mathrm{p}<.01)$ (Table 4$)$. At Week 13, post-program, no-one scored as severe/extremely severe on depression. One woman scored as extremely severe on anxiety and one scored as severe on stress (Table 3). From baseline to Week 13 , only the improvement in anxiety was statistically significant $(p<.05)$ (Table 4$)$.

\section{Experience of the Arabic Mindfulness CD}

Analysis of participants' log book entries over the program revealed how the women grew in their understanding of mindfulness concepts and mastered the different techniques, although it was not always easy; how they dealt with painful memories; how mindfulness practice assisted them in their daily lives; and how they related mindfulness to Islam, integrating it with their daily religious practices such as prayer and reflection. The same four themes were evident in comments made by participants at the group sessions and speaking individually with the facilitator after a session or at follow up. Each theme is illustrated with quotations below.

\section{Challenges in Understanding and Applying Mindfulness}

Some participants struggled with understanding concepts such as The Observing Self. The notions of self-compassion and self-care, though novel for many, were perceived as consistent with Islam. During the group discussions, participants often quoted from the Quran (chapter:verse), e.g. Prophet Noah "Oh Lord, forgive me and my parents and ..." (71:28), and
Table 3 DASS21 scores for intervention and wait-list control groups over time

\begin{tabular}{|c|c|c|c|c|c|c|c|c|c|c|}
\hline \multirow[t]{3}{*}{ Subscale } & \multicolumn{4}{|c|}{ Intervention group $(n=12)$} & \multicolumn{6}{|c|}{ Wait-list control group $(\mathrm{n}=8)$} \\
\hline & \multicolumn{2}{|c|}{ Baseline } & \multicolumn{2}{|c|}{ Week 5} & \multicolumn{2}{|c|}{ Baseline } & \multicolumn{2}{|c|}{ Week 5} & \multicolumn{2}{|c|}{ Week 13} \\
\hline & $\mathrm{n}$ & $\%$ & $\mathrm{n}$ & $\%$ & $\mathrm{n}$ & $\%$ & $\mathrm{n}$ & $\%$ & $\mathrm{n}$ & $\%$ \\
\hline \multicolumn{11}{|l|}{ Depression } \\
\hline Normal $(0-4)$ & 1 & 8 & 6 & 50 & 4 & 50 & 2 & 25 & 4 & 50 \\
\hline Mild (5-6) & 0 & 0 & 3 & 25 & 0 & 0 & 2 & 25 & 1 & 13 \\
\hline Moderate $(7-10)$ & 4 & 3 & 3 & 25 & 0 & 0 & 1 & 13 & 3 & 38 \\
\hline Severe $(11-13)$ & 2 & 17 & 0 & 0 & 0 & 0 & 3 & 38 & 0 & 0 \\
\hline Extremely Severe (14+) & 5 & 42 & 0 & 0 & 4 & 50 & 0 & 0 & 0 & 0 \\
\hline \multicolumn{11}{|l|}{ Anxiety } \\
\hline Normal $(0-3)$ & 2 & 17 & 7 & 58 & 2 & 25 & 4 & 55 & 4 & 50 \\
\hline Mild (4-5) & 0 & 0 & 2 & 17 & 0 & 0 & 1 & 13 & 1 & 13 \\
\hline Moderate (6-7) & 0 & 0 & 0 & 0 & 2 & 25 & 1 & 13 & 2 & 25 \\
\hline Severe (8-9) & 1 & 8 & 1 & 8 & 0 & 0 & 1 & 13 & 0 & 0 \\
\hline Extremely Severe (10+) & 9 & 75 & 2 & 17 & 4 & 50 & 1 & 13 & 1 & 13 \\
\hline \multicolumn{11}{|l|}{ Stress } \\
\hline Normal $(0-7)$ & 0 & 0 & 8 & 67 & 3 & 38 & 4 & 50 & 4 & 50 \\
\hline Mild (8-9) & 1 & 8 & 1 & 8 & 1 & 13 & 0 & 0 & 2 & 5 \\
\hline Moderate $(10-12)$ & 2 & 17 & 3 & 25 & 0 & 0 & 1 & 13 & 1 & 13 \\
\hline Severe (13-16) & 3 & 25 & 0 & 0 & 3 & 13 & 3 & 38 & 1 & 13 \\
\hline Extremely Severe $(17+)$ & 6 & 50 & 0 & 0 & 1 & 38 & 0 & 0 & 0 & 0 \\
\hline
\end{tabular}


Prophet Ibrahim "O Our Lord! Grant me protection and my parents and..." (14:41). One woman, an Iraqi refugee, commented:

"The idea of letting the self precede others is not selfishness and is mentioned in the Quran." (No. 9)

At first, it was difficult to maintain focus during the mindfulness exercises. Another participant, also an Iraqi refugee, and recently diagnosed with cancer, noted:

"I found it hard to focus and palpitations developed. Also I found Leaves on Stream difficult as my thoughts were scattered." (No. 6)

Table 4 Change in DASS21 scores from baseline to Week 5 for the intervention and wait-list control groups, and from baseline to Week 13 for the wait-list control group

\begin{tabular}{llll}
\hline Subscales & M (SD) & Median & $\frac{\text { Sign test for pre-post change }}{\mathrm{z}}$ \\
\cline { 3 - 3 }
\end{tabular}

\section{Intervention group $(\mathrm{n}=\mathbf{1 2})$}

\section{Depression}

Baseline$$
11.9(5.2) \quad 12.5
$$

$-3.46$

.000

Week 5

$$
4.3(2.5) \quad 4.5
$$

Anxiety

Baseline

$11.5(5.5) \quad 13.0$

$-2.89$

Week 5

$4.3(4.1) \quad 2.5$

Stress

Baseline

$15.8(4.0) \quad 16.50$

$-3.46$

Week 5

$6.7(3.3) \quad 6.0$

Wait-list control group $(\mathbf{n}=8)$

\section{Depression}

\begin{tabular}{lllll} 
Baseline & $10.3(7.6)$ & 9.5 & -1.13 & .128 \\
Week 5 & $7.3(4.1)$ & 6.5 & & \\
Anxiety & & & & \\
Baseline & $9.4(6.7)$ & 8.5 & -2.65 & .004 \\
Week 5 & $5.0(4.8)$ & 4.0 & & \\
Stress & & & & .240 \\
Baseline & $11.3(6.1)$ & 12.0 & -0.71 & \\
Week 5 & $9.9(4.3)$ & 9.5 & & \\
Wait-list control group after intervention (n=8) & \\
Depression & \multicolumn{5}{l}{. } & & \\
Baseline & $10.3(7.6)$ & 9.5 & -1.41 & .079 \\
Week 13 & $4.6(3.2)$ & 4.5 & & \\
Anxiety & & & & \\
Baseline & $9.4(6.7)$ & 8.5 & -1.89 & \\
Week 13 & $4.5(3.5)$ & 4.0 & & \\
Stress & & & & \\
Baseline & $11.3(6.1)$ & 12 & -1.13 \\
Week 13 & $7.8(3.3)$ & 7.0 & & \\
\hline
\end{tabular}

\section{Dealing with Painful Memories and Trauma}

The mindfulness exercises often brought back painful memories from their past. This lessened with continued practice as illustrated in successive log entries by one participant:

"Bad thoughts controlled my mind. I surrendered to my thoughts and stopped listening to the tracks." (No. 10, week 2)

"I forced myself this week to focus. I sat in my room with lights out and listened to the tracks. Bad thoughts started coming into my mind and I felt bodily pains. I thought why am I feeling these pains? Every time I focussed on the tracks the pain would go but then returns. My focus reached $80 \%$ and I felt that I transferred from one place to another. I was happy, as for the first time I felt relaxed." (No. 10, week 3 )

"I became used to the relaxation exercise. I am able to be mindful, not thinking of the past, and controlling my bad emotions." (No. 10, week 5)

While listening to the CD tracks some women experienced physical pain, palpitations or extreme tiredness associated with their emotional pain. Again, this generally reduced with practice.

"I couldn't cope with the Leaves on [a] Stream track. I broke down in tears when trying to place photos on leaves. I felt physically tired and severe pain in my neck when listened to The Observing Self." (No. 11, week 3)

\section{Assistance with Daily Lives}

Despite the struggle, mindfulness practice during the group sessions and listening to the CD at home bought immediate benefits which participants then found ways to maximise.

"CD less effective due to feeling of time rushed and thoughts. Played after hot shower - relaxed. Excellent!" (No. 8)

"I practise breathing skills every day.” (No. 13)

In addition to dealing with painful memories and trauma, participants learnt strategies and techniques to deal with stress and worry generally — not judging, maintaining focus and being more accepting. The techniques helped participants relax more, worry less, gain control over their thoughts, allow forgiveness in their lives, and led to improved relationships with children and grandchildren. Group sessions also assisted with setting goals and self-development. 
"The Observing Self helped me to be more in peace with myself and not blame myself as I usually do. It made be less judgemental with myself and others." (No. 2)

"Reaching the stage where we pray for those who harmed us and forgive them." (No. 16)

Some women noted how they were able to integrate mindfulness into their everyday activities, e.g. being more focused in their prayer or more mindful of eating. This did not necessarily mean that they stopped using the $\mathrm{CD}$, which for some became a core feature and enjoyable ritual of their day. After three weeks one woman noted:

"I yearn for practising the exercise in the morning as if I have a rendezvous with a beloved." (No. 9)

The following feedback on the program as a whole, and its impact, was provided to the facilitator over the phone several months later.

"I learnt so much from the Arabic Mindfulness course $C D$. Attending the classes and listening to the CD tracks have had a very positive impact on my life. I keep the CD in my car and I listen to it almost every day. I go early to pick up my children from school and sit in the car and listen to different tracks while I am waiting for them. Being mindful, to me it means being aware of my emotions and thoughts and choose to react wisely. I am particularly aware of judging and I take much more time before I jump into conclusions.

I continue to listen to the CD particularly when I am distressed and need to put things into the right perspective. When I listen to the CD I feel I am in a completely different world away from the world of distress and worries.

Being mindful changed my behaviours at home and helped improve my relationship with my children. I am calmer, less reactive and more attentive." (No. 7)

\section{Religious Significance}

Mindfulness concepts resonated with all the participants and gained significance through their religious understanding. Many referred to the Quran and the importance of mindfulness to Islamic practice as it assisted to maintain focus during prayer and recitations. One participant told the facilitator afterwards:

"Most important point for me is that mindfulness relates to my religion. For example, prayers require the person to be mindful. My mind used to wander during prayers.
Mindfulness helped me control my thoughts and focus

on any task." (No. 9)

Another woman reported:

"Mindfulness has strengthened my faith and reduced stress." (No. 18)

\section{Social Considerations}

The group sessions, which were structured around the CD, provided a source of ongoing motivation and support, including peer support. Although from different countries and backgrounds, the women had much in common and were able to relate to each other's experiences with understanding and empathy. Additionally, the groups were an opportunity to clarify and revisit concepts, such as The Observing Self, and the association between physical and emotional pain. The bilingual psychologist/group facilitator built rapport with participants and gained their trust. She maintained contact with participants who could not attend all sessions phoning them to see how they were, and monitored those who were having a difficult time. Following the program, some of the women continued to meet and support each other.

Comments in the participants' log books and feedback to facilitator indicated that they shared the mindfulness CDs and techniques with others, with benefits to their families and the broader community. Some reported sending the CD to family and friends overseas.

"[Participation in the mindfulness program] motivated me to encourage my family to take part in educational programs that train the self to face the burdens of life." (No. 9)

"We hope that these important exercises and sessions continue as they bring comfort and happiness, eliminate bad thoughts and solve personal problems easily." (No. 17)

\section{Discussion}

This study confirms the results of the earlier study in which the Arabic Mindfulness CD was shown to be culturally acceptable and effective in reducing levels of psychological distress, depression, anxiety and stress when provided to individuals as a self-management resource (Blignault et al., 2019). As in the earlier study, baseline scores on all DASS21 subscales were elevated; even more so for anxiety. This was to expected given that most of the female Muslim participants were relatively recent arrivals from Iraq and other war-torn countries 
(the program was delivered during the peak of Syrian refugee settlement in Australia) and dealing with ongoing trauma and loss as well as settlement issues (Slewa-Younan et al., 2015; Slewa-Younan et al., 2017). Since the September 11 (9/11) terrorist attacks in the United States in 2001, life has not been easy for Muslims in Australia (Khawaja \& Khawaja, 2016a) and other Western countries (Khawaja \& Khawaja, 2016b).

At Week 5, following completion of the group mindfulness program, the 12 participants in the intervention group showed clinically and statistically significant improvement, with a reduction in the number reporting severe/extremely severe levels of depression, anxiety and stress. The eight participants in the wait-list control group also showed improvement; with exception of anxiety however, the results were not statistically significant. We speculate that the reduction in anxiety may be due to reassurance as a result of attending the information session. Although participants in the wait-list control group showed further improvement post-program (Week 13), again only the result for anxiety was statistically significant. A bigger sample size would have increased the statistical power to detect true differences. Group facilitators observed a positive shift in emotions in both groups over the course of the program. Mindfulness practice helped the women to manage painful memories and the stresses of everyday living, led to improved family relationships, and enabled more focussed religious practice; skills which they readily applied to other areas of their lives. Participant feedback at the combined follow-up session (Week 14) and follow-up phone calls several months later suggested that they continued to accrue the benefits. In the earlier study, almost all of the 70 participants continued to use the CD beyond the 5 weeks, with improvements on the DASS21 subscales and the Kessler Psychological Distress Scale (K10) being maintained over time (Blignault et al., 2019).

Beyond the more common psychological conditions, there is emerging evidence of the efficacy of MBIs in the treatment of trauma-exposed populations, mostly military veterans (Boyd et al., 2018; Lang, 2017). This small study with Arabic-speaking women in Australia suggests that mindfulness may be valuable in helping refugee and migrant populations deal with trauma.

Cultural acceptability was demonstrated in the high levels of engagement and program adherence. Of the 27 women who attended the information session, 21 participated in the program and all but one of them completed it. Reasons for nonparticipation related to travelling overseas (also the case for the one dropout) and group times clashing with English classes. Participants readily related mindfulness concepts to the Quran and the teachings of the Prophets. In other MBI studies, conflict with participants' religious or spiritual beliefs has been a reason for attrition (DeLuca et al., 2018). All participants attended at least three group sessions and logged home practiced at least three weeks. That the women were getting a sufficient 'dose' of mindfulness to make a difference to/ impact on their sense of wellbeing is supported by the comments in their log sheets. Three times a week or more seems to be an effective dose for mindfulness practice (Perich et al., 2013). Questionnaire responses and comments made during the program and at follow up confirmed that the participants found mindfulness compatible with their existing cultural and religious practices and that it fitted in with their way of life. They shared the $\mathrm{CD}$ with family and friends, including those overseas.

This ongoing program of research (Blignault et al., 2019, 2021), now in its fourth phase, demonstrates the value of a community-based mental health and wellbeing support for refugees (Slewa-Younan et al., 2018). The Mindfulness Program for Arabic-Speaking Women was delivered at a venue that was non-stigmatising and accessible. The intervention was free and child care was provided. Structured around the $\mathrm{CD}$, the program was developed and delivered by an experienced female psychologist who brought a trauma-informed approach. Introduction of the unfamiliar mindfulness concepts and techniques was carefully timed so that participants developed enough skills to eventually deal with painful memories. A female multicultural health worker assisted with recruitment, program delivery and evaluation. The groups provided an emotionally-safe environment to share challenging experiences and ongoing motivation and support, while regular home practice reinforced the new skills. As in subsequent group mindfulness programs delivered to Arabic and Bangla-speaking communities in Sydney (Blignault et al., 2021), content included elements of community education and mental health literacy.

\section{Study Strengths and Limitations}

The mixed-methods evaluation was designed as a replication and extension of an earlier study that showed improvement post-program and at follow-up; with the inclusion of a waitlist control group and a different delivery mode, Arabicspeaking population and setting. The DASS21 is widely used as a measure of mental health outcomes (b; Lovibond \& Lovibond, 1995a) and has been validated with Arabicspeakers (Moussa et al., 2016). Collection of qualitative data shed light on the therapeutic process and supported interpretation of the findings. Participant engagement and program adherence were high. Study limitations include the small sample size, with only eight participants in Group 2, and lack of follow-up assessment on the DASS21. Reliance on selfreport for mental health outcomes and mindfulness home practice is another limitation. Respecting cultural sensitivities, only women were recruited. As with any volunteer sample, there may be self-selection bias; participants were open to learning new ways to improve their wellbeing through mindfulness. 


\section{Conclusions}

This study offers further support for the clinical utility and cultural acceptability of the Arabic Mindfulness CD, and for the cultural adaptation of evidence-based psychological interventions more generally. Thoughtful implementation of linguistically and culturally-tailored programs can optimise engagement and outcomes for vulnerable and under-served communities in a multicultural society. The current findings are particularly relevant to the Australian context given the large number of recent arrivals from the Middle East and North Africa on humanitarian visas (ABS, 2020). Further research is required to investigate the effectiveness of MBIs for Arabic-speaking men with refugee-like backgrounds, and the transferability of this intervention model to other minority communities.

Acknowledgements Hanan Youseff, Bilingual Multicultural Health Worker; Klara Takas for project coordination; Leissa Pitts and Eman Baghdadi for site coordination and community engagement; Fatima Hamdan for translation assistance; and Arshdeep Kaur for research assistance. Special thanks to the program participants for sharing their experiences.

Code Availability Not applicable.

Authors' Contributions LW, HS and IB conceived and designed the evaluation. HS delivered the intervention and acquired the data. IB conducted the statistical analysis and $\mathrm{CO}$ the qualitative analysis. All authors contributed to data interpretation. IB drafted the manuscript, which was edited and approved by all authors.

Funding This project was funded by South Eastern Sydney Local Health District Multicultural Health Service, with in-kind support from the project partners: South Eastern Sydney Local Health District Mental Health Service, Illawarra Shoalhaven Local Health District Multicultural Health Service and Illawarra Multicultural Services.

Data Availability The data sets are not publicly available as they contain information that could potentially re-identify individuals, but are available from LW, author upon reasonable request and with relevant ethical approval. Program materials are available from HS, author.

\section{Declarations}

Ethics Approval Ethics approval was obtained from the SESLHD Human Research Ethics Committee [HREC/14/155, with external recognition from Western Sydney University Human Research Ethics Committee [RH11768].

Consent to Participate Informed consent was obtained from all participants.

Consent for Publication Not applicable.

Conflicts of Interest/Competing Interests Not applicable.
Open Access This article is licensed under a Creative Commons Attribution 4.0 International License, which permits use, sharing, adaptation, distribution and reproduction in any medium or format, as long as you give appropriate credit to the original author(s) and the source, provide a link to the Creative Commons licence, and indicate if changes were made. The images or other third party material in this article are included in the article's Creative Commons licence, unless indicated otherwise in a credit line to the material. If material is not included in the article's Creative Commons licence and your intended use is not permitted by statutory regulation or exceeds the permitted use, you will need to obtain permission directly from the copyright holder. To view a copy of this licence, visit http://creativecommons.org/licenses/by/4.0/.

\section{References}

Australian Bureau of Statistics. (2017). Cultural diversity in Australia, 2016. In: Census of Population and Housing: Australia Revealed, 2016. https://www.abs.gov.au/ausstats/abs@.nsf/Latestproducts/ 2024.0Main\%20Features22016? opendocument\&tabname $=$ Summary \&prodno $=2024.0 \&$ issue $=2016 \&$ num $=\&$ view $=$

Australian Bureau of Statistics. (2020). Characteristics of recent migrants: Data about migrants arriving in the last 10 years including employment outcomes relating to visa type, birth country, education and language skills. People and Communities. https://www.abs. gov.au/statistics/people/people-and-communities/characteristicsrecent-migrants/latest-release\#overview.

Baer, R. (2015). Ethics, values, virtues, and character strengths in mindfulness-based interventions: A psychological science perspective. Mindfulness, 6, 956-969. https://doi.org/10.1007/s12671-015-0419-2.

Blignault, I., Saab, H., Woodland, L., \& Comino, E. (2019). Evaluation of the acceptability and clinical utility of an Arabiclanguage mindfulness CD in an Australian community setting. Transculatural Psychiatry, 56(3), 552-568. https://doi.org/10.1177/ 1363461519825880

Blignault, I., Saab, H., Woodland, L., Mannan, H. \& Kaur A. (2021). Effectiveness of a community-based group mindfulness program tailored for Arabic and Bangla-speaking migrants. International Journal of Mental Health Systems 15, 32. https://doi.org/10.1186/ s13033-021-00456-0.

Boyd, J. E., Lanius, R. A., \& McKinnon, M. C. (2018). Mindfulness-based treatments for posttraumatic stress disorder: A review of the treatment literature and neurobiological evidence. Journal of Psychiatry \& Neuroscience, 43(1), 7-25. https://doi.org/10.1503/jpn.170021.

Creswell, J. D. (2017). Mindfulness interventions. Annual Review of Psychology, 68, 491-516. https://doi.org/10.1146/annurev-psych042716-051139.

Damra, J. K., Nassar, Y. H., \& Ghabri, T. M. F. (2014). Trauma-focused cognitive behavioral therapy: Cultural adaptations for application in Jordanian culture. Counselling Psychology Quarterly, 27(3), 308323. https://doi.org/10.1080/09515070.2014.918534.

DeLuca, S., Kelman, A., \& Waelde, L. (2018). A systematic review of ethnoracial representation and cultural adaptation of mindfulnessand meditation-based interventions. Psychological Studies, 63(2), 117-129. https://doi.org/10.1007/s12646-018-0452-z.

Green, J., \& Thorogood, N. (2018). Qualitative methods for Health Research. Sage Publications.

Güldal, Ș., \& Satan, A. (2020). The effect of mindfulness based psychoeducation program on adolescents' character strengths, mindfulness and academic achievement. Current Psychology. https://doi.org/10.1007/s12144-020-01153-w.

Howarth, A., Jared, G., Smith, J. G., Perkins-Porras, L., \& Ussher, M. (2019). Effects of brief mindfulness-based interventions on health- 
related outcomes: A systematic review. Mindfulness, 10, 19571968. https://doi.org/10.1007/s12671-019-01163-1.

Janssen, M., Heerkens, Y., Kuijer, W., van der Heijden, B., \& Engels, J. (2018). Effects of mindfulness-based stress reduction on employees' mental health: A systematic review. PLoS One, 13(1), e0191332. https://doi.org/10.1371/journal.pone.0191332.

Jupp, J. (2001). The Australian people: An encyclopedia of the nation, its peoples and their origins. Cambridge University Press.

Kabat-Zinn, J. (2003). Mindfulness-based interventions in context: Past, present, and future. Clinical Psychology: Science and Practice, 10(2), 144-156. https://doi.org/10.1093/clipsy.bpg016.

Kayrouz, R., Dear, B. F., Johnston, L., Keyrouz, L., Nehme, E., Laube, R., \& Titov, N. (2015). Intergenerational and cross-cultural differences in emotional wellbeing, mental health service utilisation, treatment-seeking preferences and acceptability of psychological treatments for Arab Australians. International Journal of Social Psychiatry, 61(5), 484-491. https://doi.org/10.1177/ 0020764014553004.

Khawaja, N. G., \& Khawaja, S. (2016a). Acculturative issues of Muslims in Australia. Journal of Muslim Mental Health, 10(2), 43-53. https:// doi.org/10.3998/jmmh.10381607.0010.203.

Khawaja, N. G., \& Khawaja, S. (2016b). Acculturation of the Muslims settled in the west. Journal of Muslim Mental Health, 10(1), 3-11. https://doi.org/10.3998/jmmh.10381607.0010.102.

Lang, A. J. (2017). Mindfulness in PTSD treatment. Current Opinion in Psychology, 14, 40-43. https://doi.org/10.1016/j.copsyc.2016.10.005.

Lovibond, P. F., \& Lovibond, S. H. (1995a). The structure of negative emotional states: Comparison of the depression anxiety stress scales (DASS) with the Beck depression and anxiety inventories. Behaviour Research and Therapy, 33(3), 335-343. https://doi.org/ 10.1016/0005-7967(94)00075-u.

Lovibond, S. H., \& Lovibond, P. F. (1995b). Manual for the depression anxiety stress scales (2nd ed.). Psychology Foundation of Australia.

McDonald, R., \& Steel, Z. (1997). Immigrants and mental health. An epidemiological analysis. Transcultural Mental Health Centre.

Minas, H., Kakuma, R., Too, L. S., Vayani, H., Orapeleng, S., PrasadIldes, R., Turner, G., Procter, N., \& Oehm, D. (2013). Mental health research and evaluation in multicultural Australia: Developing a culture of inclusion. International Journal of Mental Health Systems, 7(1), 23. https://doi.org/10.1186/1752-4458-7-23.

Mirdal, G. M. (2012). Mevlana Jalal-ad-Din Rumi and mindfulness. Journal of Religion and Health, 51(4), 1202-1215. https://doi.org/ 10.1007/s10943-010-9430-z.

Moussa, M. T., Lovibond, P. F., Laube, R., \& Megahead, H. A. (2016). Psychometric properties of an Arabic version of the depression anxiety stress scales (DASS). Research on Social Work Practice, 27(3), 375-386. https://doi.org/10.1177/1049731516662916.

Perich, T., Manicavasagar, V., Mitchell, P. B., \& Ball, J. R. (2013). The association between meditation practice and treatment outcome in mindfulness-based cognitive therapy for bipolar disorder. Behaviour Research and Therapy, 51(7), 338-343. https://doi.org/10.1016/j. brat.2013.03.006.

Pigni, A. (2010). A first-person account of using mindfulness as a therapeutic tool in the Palestinian territories. Journal of Child and Family Studies, 19(2), 152-156. https://doi.org/10.1007/s10826-009-9328-0.

Rosales, A., \& Tan, S. (2017). Mindfulness-based cognitive therapy (MBCT): Empirical evidence and clinical applications from a Christian perspective. Journal of Psychology and Christianity, 36(1), 76-82.

Russell, L., Ugalde, A., White, V., Pitcher, M., Jorgenssen, S., Whitfield, K., Rasmussen, B., \& Livingston, P. (2019). Relevance of mindfulness practices for culturally and linguistically diverse cancer populations. Psycho-oncology, 28(11), 2250-2252. https://doi.org/ 10.1002/pon.5221.

Silove, D. M. (2003). Mental health of asylum seekers: Australia in a global context. In P. Allotey (Ed.), The health of refugees (pp. 6883). Oxford University Press.

Slewa-Younan, S., Mond, J. M., Bussion, E., Melkonian, M., Mohammad, Y., Dover, H., Smith, M., Milosevic, D., \& Jorm, A. F. (2015). Psychological trauma and help seeking behaviour amongst resettled Iraqi refugees in attending English tuition classes in Australia. International Journal of Mental Health Systems, 9, 5. https://doi.org/10.1186/1752-4458-9-5.

Slewa-Younan, S., Yaser, A., Uribe Guajardo, M. G., Mannan, H., Smith, C. A., \& Mond, J. M. (2017). The mental health and help-seeking behaviour of resettled afghan refugees in Australia. International Journal of Mental Health Systems, 11, 49. https://doi.org/10.1186/ s13033-017-0157-z.

Slewa-Younan, S., Blignault, I., Renzaho, A., \& Doherty, M. (2018). Community-based mental health and wellbeing support for refugees: An evidence check rapid review brokered by the sax institute for the NSW Ministry of Health, 2018. In: Evidence Check Library. Sax Institute. https://www.saxinstitute.org.au/publications/ community-based-mental-health-wellbeing-support-refugees

Steel, Z., McDonald, R., Silove, D., Bauman, A., Sandford, P., Herron, J., \& Minas, I. H. (2006). Pathways to the first contact with specialist mental health care. Australian and New Zealand Journal of Psychiatry, 40(4), 347-354. https://doi.org/10.1080/j.1440-1614. 2006.01801.x.

Thomas, J., Furber, S., \& Grey, I. (2017). The rise of mindfulness and its resonance with the Islamic tradition. Mental Health, Religion \& Culture, 20(10), 973-985. https://doi.org/10.1080/13674676.2017. 1412410.

Thomas, J., Raynor, M., \& Bakker, M. (2016a). Mindfulness-based stress reduction among Emirati Muslim women. Mental Health, Religion \& Culture, 19(3), 295-304. https://doi.org/10.1080/13674676.2016. 1168389.

Thomas, J., Raynor, M., \& Bahussain, E. (2016b). Stress reactivity, depressive symptoms and mindfulness: A gulf Arab perspective. International perspectives in psychology: Research, practice, consultation, 5(3), 156-166. https://doi.org/10.1037/ipp0000055201621448-001.

Tobin, M. (2000). Developing mental health rehabilitation services in a culturally appropriate context. Australian Health Review, 23(2), 177-184. https://doi.org/10.1071/AH000177.

Trammel, R. C. (2015). Mindfulness as enhancing ethical decisionmaking and the Christian integration of mindful practice. Journal of the North American Association of Christians in Social Work, 42(2), 165-177.

Youssef, J., \& Deane, F. P. (2006). Factors influencing mental-health help-seeking in Arabic-speaking communities in Sydney, Australia. Mental Health, Religion \& Culture, 9(1), 43-66. https:// doi.org/10.1080/13674670512331335686.

Van Dam, N. T., Van Vugt, M. K., Vago, D. R., Schmalzl, L., Saron, C. D., Olendzki, A., Meissner, T., Lazar, S. W., Kerr, K. E., Gorchov, J., Fox, K. C. R., Field, B. A., Britton, W. B., Brefcczynski-Lewis, J. A., \& Meyer, D. E. (2018). Mind the hype: A critical evaluation and prescriptive agenda for research on mindfulness and meditation. Perspectives on psychological Science, 13(1), 36-61. https://doi. org/10.1177/1745691617709589.

Publisher's Note Springer Nature remains neutral with regard to jurisdictional claims in published maps and institutional affiliations. 